\title{
Preface: what's next? COVID-19 as a planetary inflection point for places, people, policy and research
}

Academic books have many origins and often result from research and funding arising from scholars gathering, sharing, and debating thoughts in dedicated intellectual arenas. Such debates used to materialize in physical environments and develop from slowly cultivated seeds of ideas. This book is unique in having none of the luxuries of the above. The genesis of this work arose from the sudden (and then thought to be short and sharp) shock of the UK entering its first phase of lockdown back in March 2020 and the world starting to fight a global pandemic. Our collaborative space was Zoom; the development and compilation of this book was achieved completely virtually, symbolizing the shift towards the socially distant environment into which we have found ourselves living and still are as of early 2021.

The COVID-19 pandemic generated very distinct reactions from the academic community; while medicine and public health experts had no choice but to immediately be at the forefront of developing new insights and expertise about the new coronavirus, social scientists had more options. Some quite firmly refused to engage with what they considered to be a fleeting event not directly linked to their research (and to do so, would be opportunistic). Others, including the team who drove this edited collection, saw it as a turning point, due to its unprecedented nature, for research, for economies, societies and for all of us as individuals experiencing for the first time in our lives a global pandemic. While writing this preface in January 2021, all continents across the world are still affected by various forms of lockdowns, with new variants emerging and cases still rising. The pandemic is far from being over and the months and years to come will be characterized by significant unknowns, uncertainties, and challenges to which few responses are available as yet. No matter our individual trajectories, or our global context, what citizens and researchers have experienced in 2020-21 will significantly impact and change them. This is unprecedented and as such constitutes an inflection point.

Such an inflection point is related to two major transformations. First, COVID-19 triggered unparalleled upheavals. Societies, economies, policy were to date mostly constructed on planned and fixed decisions based on 
what we thought we knew about the long-term picture. Such certainties were deeply uprooted, at micro, meso, and macro levels. The pandemic made us face the unknown on an everyday basis and required constant adaptation and improvisation. Decisions were thus shaped based on weaker and more fluid rationales resulting from recent and constantly adjusted data, with little scope for longer-term planning and anticipation. Planning became an exercise in rapid improvisation. Second, COVID-19 transformed the relationship between policy and knowledge and expertise. New knowledge and expertise were needed immediately, and had to be developed, validated, and communicated at high speed. New ways of reaching individuals had to be found, particularly towards precautionary measures that had to be implemented. New landscapes of knowledge emerged and new boundary spanning networks were constructed to support the spread of this knowledge. Pandemic relevant knowledge was, still is, and will remain critical. Lessons will need to be learnt from the pandemic and this involves recording and analyzing what has been experienced by places, people, and policy, in various contexts, during the first year of the pandemic. The need to document and explore the impacts and responses of living with a pandemic are core to the genesis of this book.

This book constitutes a key contribution, amongst many others which will follow, to capturing the fast-moving landscape of knowledge that has been constructed during, and because of, the COVID-19 pandemic. In March 2020, just weeks into the pandemic hitting Europe the contract for this book was signed with Edward Elgar Publishing and contributors were approached. This as such is exceptional and denotes the unprecedented nature of what we have been experiencing to date. All contributors, were asked to: (1) explore the diversity of impacts and responses to COVID-19 in exceptionally short timeframes, including exploring immediate impacts on people, households, communities, businesses, and nations and (2), to identify and evaluate mitigation and adaptation strategies of individuals, households, communities, businesses, and nations over a longer period. We were very aware that current responses to the pandemic made original research difficult. The expectation was that each chapter was going to highlight some aspects of the pandemic and different responses based on an analysis, or reading, of an ongoing event that contributors were all experiencing.

This book was produced in less than a year and we are extremely grateful to our colleagues, friends, and acquaintances for responding positively and proactively to our requests and embarking with us on this adventure. This book is probably the first step into this interdisciplinary, intersectoral, multi-level interrogation of the pandemic; it will certainly be useful to reflect in a couple of years on these preliminary interpretations and re-visit what was said about the impacts of COVID-19 on places, people, and policy and whether they have come to pass. 
We would like to end with a reflection from the French philosopher Bruno Latour that comes from his latest book Où suis-je which can be translated as Where am I? (Empecheurs, 2021). While pointing out that States and individuals are all seeking to relax lockdown restrictions and turn themselves to recovery strategies, he notes that wider lessons should be drawn for those that he calls the 'terrestres' (i.e. those who live on Earth and recognize what this planet offers). The 'terrestres' should see the pandemic as a way to finally recognize and acknowledge what our planet is offering in the context of the climate emergency. This is a very important point which highlights the fragility of our life on earth.

As we move beyond the pandemic it is important to connect the current global pandemic with the crisis that is global climate change. The pandemic has revealed that people, organizations, places, and governments are able to engage in rapid improvisation in response to an immediate crisis. Now is the time to mitigate and adapt to climate change and for all to recognize that climate change is a much more serious threat to life on earth as we know it than COVID-19. The key is to place people first and to focus in particular on younger and future generations.

Lauren Andres, John R. Bryson, Aksel Ersoy and Louise Reardon 31 January 2021 